\title{
Environment, Equivalence Inferences, and the Twins: A Reply to Sober
}

\author{
T.Y. William Wong*
}

\begin{abstract}
Elliott Sober (2020) can be understood as advancing two distinct arguments that similarly conclude that evolutionary theory does not say that Scriven's (1959) infamous twins have the same fitness, despite the twins' identical genotypes and phenotypes. The first argument relies on denying that evolutionary theory can say that the twins are in the same environment, and the second relies on asserting an epistemic access asymmetry between token fitness and trait fitness. Motivated by good reasons, I respond to both of these arguments by showing that the theory of evolution by natural selection has adequate means for determining whether the twins are in the same environment via causal-probabilistic decomposition, and that equivalence in fitness can be asserted even from a partial theory of fitness, regardless of epistemology. Finally, I point out an absurd result regarding genetic drift as indiscriminate sampling that follows if Sober's conclusion is true.
\end{abstract}

\section{Keywords}

evolutionary fitness $\bullet$ Partial Theory of Fitness $\bullet$ twins $\bullet$ Sober, Elliott $\bullet$ evolutionary environment $\bullet$ genetic drift $\bullet$ environment-type

\section{Introduction}

Within the philosophy of biology, Michael Scriven's (1959) twins has become a well-known thought experiment, due to its being the impetus for various lines of discussion concerning the contentious definition of evolutionary fitness, the distinction between natural selection and genetic drift, and evolutionary environments. As one version of the story goes: two twins who, ex hypothesi, are genotypically and phenotypically the same are located side by side on a mountain. An unfortunate event ensues whereby a lightning strike kills one of the twins, whilst the other twin survives. For added disparity, the surviving twin goes on to proliferate extensively by bearing a large number of offspring. Importantly, it is usually inferred, from the stipulations of the thought experiment, that the twins also have the same evolutionary fitness (e.g., Mills and Beatty 1979; Beatty 1984; Rosenberg 1985). The inference is important because the premise that the twins have the same fitness paves way for a number of influential views; most notably, it has led to the propensity interpretation of evolutionary fitness (Brandon 1978; Mills and Beatty 1979; Beatty and Finsen 1989).

*16/F, The Hong Kong Club Building, 3A Chater Road, Central, Hong Kong, william.wong@outlook.com

Received 14 May 2020; Revised 4 December 2020; Accepted 4 December 2020 doi:10.3998/ptpbio.16039257.0013.002

๑ OPEN ACCESS - PTPBIO.ORG 
In the paper 'Fitness and the Twins', Sober (2020) argues that evolutionary theory does not actually say that the twins have identical fitness, despite their sharing the same genotype and phenotype. In arguing for this conclusion, Sober (2020) can be understood as employing a two-pronged approach, with each prong constituting a distinct argument.

The first prong is an argument that asserts that evolutionary theory does not say that the twins are in the same environment. And if it cannot be said, by evolutionary theory, that the twins are in the same environment, then it cannot be said, by evolutionary theory, that the twins have the same fitness. This is because fitness is understood as being relativised to a particular environment (Mills and Beatty 1979; Rosenberg 1985; Shanahan 1989; Brandon 1990).

The second prong is an argument that asserts that it is epistemically difficult to ascertain individual token fitness, like that attributed to 'Arnold the armadillo'. But Sober maintains that the epistemic situation is quite the opposite for trait fitness, like that attributed to a 'leathery armoured shell'. In other words, biologists have good epistemic access to only the latter. Drawing on this epistemic asymmetry, Sober claims that evolutionary theory is concerned with trait fitness rather than token fitness, and thereby, is hard-pressed take a stand on the token fitness of either twin. But it is further claimed that, if evolutionary theory cannot take a stand on the token fitnesses of the twins, it cannot take a stand on whether the twins have the same fitness.

In the present paper, I challenge both of these arguments. My challenge is motivated, in the first instance, by the fact that the theory of evolution by natural selection, pace Sober (2020), offers quite natural means for determining whether the twins are in the same environment or, at least, the same environment-type. This is because the theory of evolution by natural selection is both a probabilistic and a causal theory (Hodge 1987; Brandon 1990). In particular, its intrinsic framework allows for the decomposition of various causal factors that matter for the recognition (and hence, delineation) of the ecological environment. This kind of thinking was the fruit of much discussion that attempted to conceptually tease apart the improbable results of natural selection from genetic drift (e.g., Hodge 1987; Millstein 2002; cf. Beatty 1984; Shanahan 1992), and I adapt it here. Accordingly, in section 2.1.2, I shall show that the twins can be shown to be in the same environment from a causal-probabilistic perspective. Secondly, my challenge is also motivated by the fact that the conclusion that Sober seeks to establish has unsettling implications for a part of evolutionary theory that philosophers of biology have, through much pain, made progress on over the last forty years. More specifically, the conclusion that evolutionary theory does not say that the twins have the same fitness would seem to imply, absurdly, that one cannot say that the twins are undergoing genetic drift as indiscriminate sampling. I shall speak to this issue in section 3, but, first, I begin with a reconstruction of Sober's (2020) arguments and my responses to them.

\section{Sober's Two-Pronged Approach}

Sober (2020) draws widely upon disparate areas in the philosophy of biology and impressively synthesises them together to advance two distinct arguments. Although Sober never explicitly describes his approach as being 'two-pronged', my reading is that, indeed, two self-contained arguments can be recognised 1 However, as a point of convergence, both arguments attempt to assert the same conclusion, namely, that evolutionary theory does not say that the twins have the same fitness.

1. This is regardless of whether Sober was aware of it or not. These arguments are said to be 'self-contained' because neither argument depends on the other, nor do they share any premises. Seemingly the only point of connection between the two is that when Sober entertains the "design analysis" (2020) as an epistemic method of ascertaining token fitness, he says that one cannot uniquely identify the environment at hand, anyways.

๑ OPEN ACCESS - PTPBIO.ORG 
One might wish to begin by addressing the elephant in the room, which is, indeed, the very large qualifier: 'evolutionary theory does not say'. Such logical circumscription weakens the conclusion greatly as the conclusion becomes conditionalised upon the content of evolutionary theory, whatever that is considered to be? As such, the conclusion does not deny that the twins have the same fitness, but specifically asserts that evolutionary theory does not say that they do. I take it that, in having this qualifier, Sober seeks to engage with the structure of evolutionary theory, itself. In particular, he is concerned with the relations between the fitness, on one hand, and, the equally theory-laden terms of environment, survival, and reproduction, on the other hand.

However, given the qualifier, one cannot help but surmise whether 'evolutionary theory' can be naturally accompanied by some auxiliary principle (be it biological, metaphysical, or otherwise) that allows for an inference to the twins' contended equality in fitness. For example, in response to the first prong, such a principle might outline the conditions in which two individuals share the same environment, whereby the twins case, ex bypothesi, might actually fulfil these conditions. Another principle might allow for the derivation of token fitness from trait fitness, such that even if evolutionary theory does not directly assign token fitnesses, they can be derived by other means. In fact, as a pre-emptive measure, Sober attempts to ward off an appeal to such principles by entertaining two propositions he terms ' $S$ ' and ' $C$ ', one of which we will come to investigate. Ultimately, Sober rejects, for one reason or another, that these principles allow for an inference to the twins' equality in fitness.

\subsection{First Prong: Denial of a Common Environment}

The first prong can be characterised as the following argument:

EP1. There is nothing in evolutionary theory that says that the twins must be in the same environment.

$E P 2$. Fitness is relativised to a particular environment.

$C 1$. There is nothing in evolutionary theory that says that the twins have the same fitness.

It should first be noted that the environment-relativity of fitness, which is asserted by way of $E P 2$, is crucial for this argument. It is crucial because the mode of attack is to deny that, according to evolutionary theory, the twins share the same environment. To this end, there is a principle that has repeatedly cropped up in discussions of fitness (e.g., Rosenberg 1978, 1985; Mills and Beatty 1979; Sober 1984; Brandon 1990; Beatty 1992). This principle was meant to theoretically underwrite the attractive idea that fitness is an ability, rather than actual success, by associating fitness to the physical properties of an individual. But it is here that the principle also serves Sober well. Not surprisingly, Sober brings forth this principle whilst making sure to flag that the 'environment' is a part of an individual's properties:

$(S)$ The fitness of token individual $i$ in environment $E$ at time $t$ supervenes on $i$ 's other properties at time $t$, including the property of living in environment $E$ (Sober 2020).

2. Firstly, theories are always in flux, and evolutionary biology is no exception. Secondly, practicing biologists and philosophers of biology actively disagree on the content of 'evolutionary theory', whereby some of these disagreements may bear on the current issue.

๑ OPEN ACCESS - PTPBIO.ORG 
It serves Sober well because the principle directly supports the premise EP2. According to the principle, the fitness of an individual depends (via a supervenience relation) on the environment in which it is situated. In this way, the principle relativises environments to fitness. Sober states that $S$ is a plausible principle and concludes that, according to the principle, if the twins' environments are merely similar, then the principle $S$ does not imply that the twins have the same fitness.

It is at this argumentative juncture that Sober questions whether a "shoulder to shoulder" $(2020,3)$ arrangement of the twins is sufficient for evolutionary theory to say that the twins are in the same environment. Importantly, Sober (2020) is not formally denying that the twins are in the same environment, but, rather, he suggests that there is no reason to think that the twins are in the same environment. He adds that even if the twins are in such an arrangement, they will still be found in different 'spatial locations' (Ibid.).

But whether a difference in spatiotemporal location is sufficient for saying that the twins are in different environments is not so obvious. Rather, this partly depends on the coarseness of our descriptions; Shanahan (1992) has made this very point in relation to environments. For example, if one were to coarsely recognise environments according to $5 \mathrm{~m}$ by $5 \mathrm{~m}$ transects, then the twins (assuming that they are ordinarily sized human twins) will surely be in the same environment. On the other hand, if the resolution is much higher, say, $0.5 \mathrm{~m}$ by $0.5 \mathrm{~m}$ transects, then the twins would likely be in different environments. Spatiotemporal locales are, of course, only a crude way of delineating environments that serves the present illustrative purposes, but the point is that there will inevitably be a matter of specifying the grain. And depending on how the grain is specified, any two environments can be the same or different. Perhaps, all of this is to add to the difficulty, which Sober points out, of determining whether the twins are, indeed, in the same environment.

However, even if Sober was right in that there is no reason to think that the twins are in the same environment such that principle $S$ fails to imply that the twins have the same fitness, the devil is in the details. That is, even if $S$ does not entail that the twins have the same fitness, there may be alternative routes for establishing that the twins do.

There might exist a similar and equally plausible principle to $S$ :

$\left(S^{\prime}\right)$ The fitness of token individual $i$ in environment $E$ at time $t$ supervenes on $i$ 's other properties at time $t$, including the property of living in environment-type $E$.

The difference between $S$ and $S^{\prime}$ is that the latter stipulates environment-types rather than token environments. However, $S^{\prime}$ might be the sort of principle that is actually more faithful to the discussions of evolutionary fitness. This is because fitness was meant to convey a sense of 'adaptive fit' with particular ecological circumstances (Millstein 2016), and ecological circumstances are best typified by environment-types, as the latter would, by definition, be fully coextensive with environments that possess the respective ecological features. This is akin to cars with blue paint being best typified by the 'blue-car-type'.

Accordingly, if $S^{\prime}$ is true, and if the twins' environments are similar enough such that they belong to the same environment-type, then $S^{\prime}$ will imply that the twins have the same fitness, mutatis mutandis. ${ }^{[}$

3. The interpretation of $E P 2$ would also change such that fitness is understood to be relativised to environmenttypes.

๑ OPEN ACCESS - PTPBIO.ORG 
What should immediately strike the reader is that there is a glaring terminological issue. Is the term 'environment' in principle $S$ supposed to refer to a token environment or environmenttype? A footnote in Sober (2020, footnote 7) sheds some light: "What matters here is whether the twins live in type-identical environments". So, if one is to interpret $S$ as being logically equivalent to $S^{\prime}$, then the problem collapses into whether the environments of the twins are 'similar enough' to be of the same type.

Indeed, this sort of question might be ordinarily encountered by a laboratory experimenter. It may be asked, by the experimenter, whether populations of $E$. coli need to be found on the same token petri dish in order to be in the same environment-type, as to satisfy the experimental condition of keeping certain variables fixed, or can they merely be located on the same type of glucose medium, which may be found on distinct petri dishes. I, for one, do not think that the experimenter will hesitate a moment in answering this question. This is because it is obvious to practicing biologists that the features (e.g., type of food source, concentration) causally relevant to $E$. coli are determined by the glucose medium rather than by which token petri dish they are in. This is to form the crux of my argument.

I shall argue that the theory of evolution by natural selection provides systematic means for determining whether two environments are of the same type. In other words, I argue that $E P 1$ is false in that evolutionary theory can say that the twins are in the same environment-type. I would, however, like to commence with a modified version of the twins thought experiment in order to prime the reader into thinking about the sort of features of the environment that are causally relevant. And after reading the modified thought experiment, if the reader is convinced that the twins in the modified thought experiment have identical fitness and that there is no evolutionarily significant difference between the modified thought experiment and Scriven's, then the reader ought to also conclude the twins in Scriven's experiment have identical fitness.

\subsubsection{Counterfactual World Twins}

We can imagine a counterfactual world in which there is a genotypically and phenotypically identical twin to an individual in a different world. In this counterfactual world, the twin is standing in the exact same spot. That is, they are no longer shoulder to shoulder as in the Scriven's case but are physically situated in the exact same spot (albeit in a counterfactual world). But, because they are standing in the exact same spot, the probability of getting struck by lightning is also the same; let us say, 0.05 in both worlds. Even though 0.05 is by no means a high figure, unfortunately, lightning strikes a twin in only one of the worlds. For added disparity, the surviving twin goes on to proliferate extensively.

Now, except for the fact of whether the lightning strikes, the worlds are entirely the same such that the twins have the same physical properties and their environment-types are the same (if not identical environments as well). Their environment-types are the same because the probability of getting struck by lightning is the same, even if it turns out that only one twin is struck by lightning. The nature of probability is such that it is possible for lightning to strike in only one of the worlds, despite the probability of lightning strikes being the same in both worlds. This can be illustrated with the case of a coin resulting in different outcomes in a series of tosses, despite its bias remaining constant between tosses. But, according to $S$ or $S^{\prime}$ (whichever you prefer), since the twins are identical and are in the same environment-type, the counterfactual twins must also have the same fitness.

This modified telling of the twins case is meant to preclude any possibility of the twins being found in different environment-types, on account of any spatiotemporal difference or otherwise. According to the stipulations of the counterfactual world case, the twins are found

๑ OPEN ACCESS - PTPBIO.ORG 
in the exact same spot of counterfactual worlds which differ in only one fact: whether lightning strikes; but, importantly, not the probability of whether lightning strikes. Thus, if one does not think that there is any evolutionarily significant difference between the counterfactual twins case and Scriven's twins case, then one must hold the same position towards the contended fitness equality of the twins in both thought experiments.

That is, if one believes that all the evolutionary factors (e.g., survival, reproduction, environment-type) are the same across both thought experiments, then one's position towards both cases should be the same. However, I suspect that Sober would like to reject the antecedent; he might, for example, assert that there is no reason to think that the twins in Scriven's case are in the same environment-type, even if the counterfactual twins are in the same environment-type.

Fortunately, twins need not go as far as to be located in the exact same spot of counterfactual worlds in order to occupy the same environment-type. The conceptual bar is set much lower. That is, there are certain features of the environment that are relevant to the reproduction and survival of individual organisms, by which one can recognise the environment at hand (Brandon 1990; Abrams 2009). The scope of my task, however, does not extend to proposing a taxonomy of which features matter. This is part of the more challenging, so-called 'problem of the reference environment' (Abrams 2009). My point is merely that the theory of evolution by natural selection has the means of recognising the environment-type along certain causal-probabilistic conditions (whatever those conditions are), and, for this reason, EP1 ought to be rejected.

\subsubsection{The Causal-Probabilistic Framework of Environments}

In this section, I shall argue that the theory of evolution by natural selection prescribes the means for recognising and delineating evolutionary environment-types. Much of this is inspired by the philosophical literature on distinguishing the improbable results of natural selection from genetic drift (e.g., Beatty 1984). The conceptual confusion has since been dissipated by Hodge (1987) and Millstein (2002), who argued that under a causal analysis, the process of natural selection is distinct from the process of genetic drift. In brief, to delineate genetic drift from natural selection, all one needs to ask is whether the survival and/or reproduction of particular individuals, but not others, in a population is owing to physical differences amongst the individuals in the population. In other words, did the properties of a sampled individual play a causal role in its differential survival or reproduction? If it did, then it was considered a case of selection, and if it did not, then it was a case of drift.

This type of case-by-case causal decomposition is useful here, as Brandon (1990) has argued that two individuals are in a common environment-type if all the causally relevant features are the same for the two individuals. Thus, one can ask of Scriven's twins, What are the causal mechanisms that would affect the twins' reproductive and survival success? and Are there are any probability differences between the two twins with respect to these causal factors?

In the case of the twins, the only stipulated event of concern was the lightning strike. And so, one ought to ask whether the probability of being struck by lightning is the same for both twins. If it is the same, then, following Brandon (1990), the twins are in the same environmenttype, at the very least. ${ }^{-1}$ Conversely, if there is a difference in probability, then the twins are not in the same environment-type.

4. The twins may also be considered to be in the same token environment since they are standing by side, but this depends on which grain of specificity is appropriate, about which I am currently neutral. 
As the matter stands, it is implied in Scriven's case that the twins have the same probability of being struck by lightning, as they are standing shoulder to shoulder. It would not be a matter of coincidence that the twins have the same probability of being struck by lightning given that they are standing to shoulder to shoulder. After all, their spatial proximity is conducive to their having the same probability of being struck by lightning.

However, I concede that, on one reading, the implication is not watertight because the case may still allow for the possibility that there happens to be some ecologically significant border that separates the two twins. And this border may be sufficient to impel different probabilities of survival. Ultimately, the underspecification of the Scriven's (1959) original case and some subsequent retellings have left it an open question whether the probability of being struck by lightning is the same for both twins. But suppose we fill in the gap and read Scriven and subsequent authors charitably, for the case's intention to hold all factors fixed, except the lightning strike, is clear. Let us interpret the twins as having the same probability of being struck by lightning. This would pull it closer to the counterfactual twins case. Accordingly, given that all the causally relevant factors (and their probabilities) are the same for both twins, they are in the same environment-type.

The situation can be made different by altering the case. Different regions of mountains have different probabilities of lightning strikes; higher altitudes are likely to have a greater probability of lightning strikes than lower altitudes. As such, if the twins case were such that one twin was found at the top of the mountain whilst the other at the bottom, then that might very well be sufficient to imply two different environment-types. This would be because the probability of being struck by lightning would be different between the two twins. So, even if they are identical in genotype and phenotype, they may not have the same fitness on account of occupying different environment-types.

The picture can be complicated further by considering other causal factors. Suppose that the mountain is subject to a volcanic eruption, whereby the probabilities of a twin perishing, due to a volcanic eruption, at the top and bottom of the mountain are the same. If lightning strikes are removed from the equation, then a twin that is at the top of the mountain can be said to be situated in the same environment-type as the twin that is at the bottom of the mountain. This is because the probability of survival is now rendered the same, given that the only causal factor relevant to survival is consistent in both regions. As such, whether the twins are in the same environment highly depends on the stipulations of the case. And the stipulation which matters is whether the twins are subject to the same complement of causal factors, with the same probabilities.

The point is that the theory of evolution of natural selection has adequate means for determining whether the twins are in the same environment-type. One merely has to ask whether the causal factors (and their probabilities) are the same. Although, as mentioned previously, identifying the full range of causal factors is part of the so-called 'reference environment problem', and can sometimes be epistemically difficult, the twins case is a thought experiment, whereby the causal factors at hand are quite clear cut. That is, as per the stipulations of the case, only the lightning strike is of concern, as whether the lightning strikes was meant to be the only independent variable. All in all, Sober's argument from denial of a common environment fails.

Moreover, there would also seem to be some odd results, if one were to invoke an extremist method of environmental delineation whereby individuals that are shoulder to shoulder are to be in different environments, necessarily ${ }^{\square}$ The conceptual bar for whether individual organisms

5. This leaves open the possibility that there are some circumstances in which two individuals standing side by side can have different probabilities of survival and/or reproduction. Perhaps, there is an ecologically significant border between the two. 
(irrespective of whether they are twins) are in a common environment-type should not be set so high as to always preclude any two organisms from occupying the same environment-type. If one's approach is to codify environments in as many dimensions, or in as fine-grained a manner, as possible in order to distinguish an environment from every other environment of another organism, then fitness would be hard-pressed to play any explanatory or predictive role ordinarily invoked of it. It would, for example, be impossible to say that there were more descendants of individual $A$ rather than descendants of individual $B$, because individual $A$ was fitter than individual $B$, since those individuals would necessarily be in different environment-types. Thus, there are also practical grounds to think that the twins can be in the same environment-type.

Nonetheless, Sober (2020) also advances a different argument that draws on the epistemology of evolutionary fitness.

\subsection{Second Prong: Denial of Token Fitness}

The second argument can be characterised by the following:

$F P 1$. There is nothing in evolutionary theory that says that evolutionary fitness is applied to token individuals rather than trait types.

FP2. Evolutionary theory does not assign token fitness to either of the twins, because evolutionary theory is about the fitness of trait types.

$C 1$. There is nothing in evolutionary theory that says that the twins have the same fitness.

A significant portion of Sober's (2020) paper is dedicated to establishing FP1 ${ }^{0}$ In support of FP1, Sober argues that there are great epistemic difficulties in ascertaining token individual fitness (with two admitted exceptions ${ }^{\text {I) }}$ ) that do not apply to ascertaining the fitness of traits. The basis of this argument is that there is an inevitably small sample size (i.e., 1) when it comes to individual token organisms, whereas trait types will be instantiated many times. And Sober claims that small sample sizes are poor evidence for determining fitness (even if maximum likelihood estimates can be obtained). Much of this is a reiteration of an earlier argument found in Sober (2013).

In further defense of $F P 1$, Sober rules out a number of ways of deriving the fitness of token individuals. He rejects, for one reason or another, that token fitnesses can be obtained from 'design analyses' (analogous to examining the makeup of a coin), optimality models, and, a principle that links token to trait fitness. Presently, I do not question Sober on these points, and I remain neutral with respect to the epistemology of fitness, and, moreover, the move from epistemic inaccessibility to FP1 (however, see Pence and Ramsey (2013)).

But the final thrust of Sober's argument is that if evolutionary theory cannot inform the token fitness of individual organisms, then evolutionary theory cannot possibly say that the twins have identical fitness. It is with this final move that I wish to contend. In other words, I shall argue that $C 1$ does not follow from $F P 2$.

6. FP1 is a premise that has also been asserted by others in the past, including Sober, himself (e.g., Ettinger, Jablonka, and Mclaughlin 1990; Sober 2020)

7. The exceptions are (i) when it is known that an individual organism possesses a lethal trait and (ii) when one can perform interpolation to estimate the fitness of an individual. 


\subsubsection{Equivalence from a (Partial) Theory of Fitness}

Whilst there is contention over how evolutionary fitness is to be defined (e.g., Brandon 1990; Rosenberg 1994; Abrams 2009; Sober 2013; Pence and Ramsey 2013; Sober 2020), there is much less contention regarding the assertion that it is dependent on survivability, reproducibility, and environment. In other words, even if it is not clear exactly how these are related to fitness, these are components that are undoubtedly a part of some function of fitness.

But the twins case stipulates that these components are variables that are kept constant amongst the two twins. That is, it is, by hypothesis, that all the factors in the twins case that account for fitness are fixed. Thus, even if one cannot attribute a value to the fitness of a token individual, it is within the capacity of a partial theory of fitness to infer that the twins have equal fitness. In other words, an equivalence of the fitness of the twins can be asserted by way of theory.

Consider an analogy. In industry, stainless steel tubes form the raw materials for many other products. Suppose that each factory must manufacture tubes at different lengths (perhaps in an attempt to avoid competition) and each factory can manufacture only one length of tube (perhaps their banding saws cannot be adjusted). When two tubes are encountered, even without measuring the length of the tubes, or if it is epistemically difficult to do so (perhaps one lacks the tools), one can still conclude whether the two tubes are of the same or different length, given enough information. For example, if one knew that two tubes came from the same factory, then one could conclude that they are of the same length, even if the actual length is not known.

The twins case is similar because as per its stipulations, the genotype, phenotype, and-as I maintained in section 2.1.2 - the environment-type are the same. To think otherwise would be unfaithful to the intentions of the twins case After all, the twins thought experiment does not attempt to illustrate the empirical accessibility of token individual fitness or trait fitness. It is simply meant to illustrate an equivalence in fitness, so that fitness can be inferred to be different from actual survival or reproductive success, thereby dissipating the tautology allegations. In this regard, it is a perfectly competent thought experiment. That is, regardless of the epistemological status of fitness, it follows from a partial theory of fitness that the twins can have identical fitness.

Now, the present paper is inclined to agree with Sober (2020) in that token fitnesses are practically and/or epistemically difficult to ascertain. However, the crucial difference between Sober (2020) and the position of the present paper is whether the twins can be said, by evolutionary theory, to have the same fitness. Sober (2020) would answer in the negative by citing a lack of information; namely, whether the twins share the same environment-type and the exact token fitnesses of the twins. In contrast, the present paper argues that evolutionary theory has what it takes to say that the twins share the same environment-type, and, moreover, it allows for inferences about the equivalence of their fitness, even without ascertaining individual token fitnesses.

\section{The Twins and Genetic Drift}

Thus far, I have argued that the theory of evolution by natural selection provides adequate means for determining whether the twins are in the same environment. This is in direct contradiction with EP1 of Sober's first argument. Regarding the second prong, I have also argued that the conclusion does not follow from $F P 2$, since equivalence statements can still be asserted without

8. Even if Scriven's case is truly under-determining and even if, for some reason, it cannot be charitably interpreted as the twins having the same environment-type, then one can simply consider a different twins case-one that is closer in form to the counterfactual twins case posed.

๑ OPEN ACCESS - PTPBIO.ORG 
epistemic access to fitness values. I would like to end this paper by way of discussing an absurd result that arises if it cannot be said that the twins have the same fitness, and this is a result that is especially relevant to Sober (2020).

Genetic drift is a heterogeneous category of disparate processes and outcomes (Beatty 1984). One notable species of drift is indiscriminate sampling with respect to physical differences (Beatty 1984; Millstein 2002). In the case of the Scriven's twins, there were presumably no physical differences that were causally relevant to why the twin that got struck by lightning was the one that was struck. That is, presumably, the lightning strike did not choose one twin over another because of some physical difference between the two. As such, this would be a case of genetic drift as indiscriminate sampling with respect to physical differences.

However, if the twins cannot be said to have the same fitness (whether it is because they cannot be said to be in the same environment or token fitnesses cannot be attributed to them), then the lightning strike can no longer be said to be an instance of drift (even if it actually is). This is because if the twins cannot be said to have the same fitness, then there remains the possibility of there being physical differences between the twins that matter for their survival. In other words, it is possible for there to be a physical property (e.g., a different environment) of the eliminated twin, but not of the surviving twin, that was causally relevant to the eliminated twin's death. As such, the lightning strike might be an instance of selection rather than drift. It is impossible to make an ascription either way.

The diagnosis is that the conditions for attributing identical fitness to the twins are the same as (or, at the very least, include) the conditions for attributing genetic drift as indiscriminate sampling; namely, that there are no physical differences between the twins that matter to survival.

However, if instances of genetic drift as indiscriminate sampling cannot be attributed to the most favourable of cases (i.e., hypothetically identical twins), then what hope is there for genetic drift as indiscriminate sampling to be attributed elsewhere? In other words, if Sober is right that evolutionary theory cannot say that the two twins-or any two individuals for that matter-have the same fitness, then evolutionary theory will lack the means to say that there are instances of genetic drift where they occur.

Moreover, this would also mean that genetic drift would no longer be the 'chance factor' that would account for how actual survival and reproductive success can deviate from expected survival and reproductive success. For example, how could one ever say that drift occurred rather than selection? After all, one cannot rule out that there was a physical property that was causally relevant to differential success. But if the lightning in the twins case was just an instance of selection, then it would imply that expected success is actual success. As such, Sober's conclusion would undermine any attempt that relies on drift to fend off the charge of tautology, including Sober's (2020) very own 'replacement argument', which he offered in closing.

This 'replacement argument' attempts to establish that fitness is not the same as actual survival and reproductive success without an appeal to the twins case. Instead, it relies on the claim that there can be differential actual success without selection due to the operation of drift. However, the contradiction arises precisely when drift cannot be said to occur, since any physical differences relevant to success between any individuals (twins or not) cannot be ruled out.

So, perhaps, the twins case is to retain its place of importance within the philosophy of biology. One cannot simply cast it aside and independently assert that drift can explain deviation in actual success from expected success. After all, it is for the same reason-namely, that the twins are physically the same in all aspects- that the twins have the same fitness and that genetic drift as indiscriminate sampling can be said to have occurred. 


\section{Acknowledgments}

I would like to thank Pierrick Bourrat, Tyler D.P. Brunet, and Hugh Desmond.

\section{Literature cited}

Abrams, Marshall. 2009. "What Determines Biological Fitness? The Problem of the Reference Environment." Synthese 166 (1): 21-40. https://doi.org/10.1007/s11229-007-9255-9.

Beatty, John. 1984. “Chance and Natural Selection.” Philosophy of Science 51 (2): 183-211. https://doi. org/10.1086/289177.

Beatty, John. 1992. “Fitness: Theoretical Contexts.” In Keywords in Evolutionary Biology, edited by Evelyn Fox Keller and Elisabeth A. Lloyd, 115-19. Cambridge, MA: Harvard University Press.

Beatty, John, and Susan Finsen. 1989. "Rethinking the Propensity Interpretation: A Peek Inside Pandora's Box." In What the Philosophy of Biology Is, edited by Michael Ruse. Springer.

Brandon, Robert N. 1978. "Adaptation and Evolutionary Theory." Studies in History and Philosophy of Science 9 (3): 181-206.

Brandon, Robert N. 1990. Adaptation and Environment. Princeton: Princeton University Press.

Ettinger, Lia, Eva Jablonka, and Peter Mclaughlin. 1990. "On the Adaptations of Organisms and the Fitness of Types." Philosophy of Science 57 (3): 499-513. https://doi.org/10.1086/289570.

Hodge, M.J.S. 1987. "Natural Selection as a Causal, Empirical, and Probabilistic Theory." In The Probabilistic Revolution, edited by L. Kruger, G. Gigerenzer, and M.S. Morgan, 2:233-70. Cambridge, MA: MIT Press.

Mills, Susan K., and John H. Beatty. 1979. "The Propensity Interpretation of Fitness.” Philosophy of Science 46 (2): 263-286. https://doi.org/10.1086/288865.

Millstein, Roberta L. 2002. “Are Random Drift and Natural Selection Conceptually Distinct?” Biology and Philosophy 17 (1): 33-53. https://doi.org/10.1023/a:1012990800358.

Millstein, Roberta L. 2016. "Probability in Biology: The Case of Fitness." In The Oxford Handbook of Probability and Philosophy, edited by Alan Hájek and Christopher Hitchcock. Oxford: Oxford University Press. https://doi.org/10.1093/oxfordhb/9780199607617.013.27.

Pence, Charles H., and Grant Ramsey. 2013. "A New Foundation for the Propensity Interpretation of Fitness.” The British Journal for the Philosophy of Science 64 (4): 851-881. https://doi.org/10.1002/ 9780470015902.a0003443.pub2.

Rosenberg, Alexander. 1978. "The Supervenience of Biological Concepts." Philosophy of Science 45 (3): 368-386. https://doi.org/10.1086/288813.

Rosenberg, Alexander. 1985. The Structure of Biological Science. Cambridge: Cambridge University Press.

Rosenberg, Alexander. 1994. Instrumental Biology, or the Disunity of Science. University of Chicago Press.

Scriven, Michael. 1959. "Explanation and Prediction in Evolutionary Theory.” Science 130 (3374): 477482. https://doi.org/10.1126/science.130.3374.477.

Shanahan, Timothy. 1989. "Beatty on Chance and Natural Selection.” Philosophy of Science 56 (3): 484489. https://doi.org/10.1086/289503. 
Shanahan, Timothy. 1992. "Selection, Drift, and the Aims of Evolutionary Theory." In Trees of Life, edited by Paul Griffiths. Australasian Studies in History and Philosophy of Science 11. Dordrecht: Springer. https://doi.org/https://doi.org/10.1007/978-94-015-8038-0_6.

Sober, Elliott. 1984. The Nature of Selection: Evolutionary Theory in Philosophical Focus. University of Chicago Press.

Sober, Elliott. 2013. "Trait Fitness Is Not a Propensity, but Fitness Variation Is." Studies in History and Philosophy of Science Part C: Studies in History and Philosophy of Biological and Biomedical Sciences 44 (3): 336-341. https://doi.org/10.1016/j.shpsc.2013.03.002.

Sober, Elliott. 2020. "Fitness and the Twins." Philosophy, Theory, and Practice in Biology 12 (1). https: //doi.org/10.3998/ptpbio.16039257.0012.001.

(C) 2020 Author(s)

This is an open-access article distributed under the terms of the Creative Commons Attribution 4.0 International license, which permits anyone to download, copy, distribute, display, or adapt the text without asking for permission, provided that the creator(s) are given full credit.

ISSN 2475-3025 